\title{
Linguistic Truth-Valued Lattice Value Propositional Logic System (P(X)
}

\author{
Jiajun Lai ${ }^{1,2}$ Kaijun $\mathrm{Xu}^{1,2}$ Yang $\mathrm{Xu}^{2}$ Zhaoyou Zeng $^{3}$ \\ ${ }^{1}$ School of Information Science \& Technology, Southwest Jiaotong University, Chengdu 610031, P. R. China \\ ${ }^{2}$ Intelligent Control Development Center, Southwest Jiaotong University, Chengdu 610031, P. R. China \\ ${ }^{3}$ School of Economics and Management, Southwest Jiaotong University, Chengdu 610031, P. R. China
}

\begin{abstract}
In the present paper, as continuous work about linguistics truth-valued LIA and its properties (CESA2006), the lattice value propositional logic system whose valuation field look as linguistic truth value LIA (briefly, L-LIA) is focused. Firstly, some properties about linguistic truth value LIA are discussed. On the other hand, some concepts about linguistic truth value lattice-valued propositional logic system $\ell \mathrm{P}(\mathrm{X})$ is established, whose truth value domain is a linguistic truth-valued lattice implication algebra, and the semantic problems of $\ell \mathrm{P}(\mathrm{X})$ are investigated.
\end{abstract}

Keywords: Linguistic truth-valued LIA, Valuation, Valid formula, $(\alpha, \beta)$-valid.

\section{Introduction}

L. A. Zadeh introduced and developed the theory of approximate reasoning based on the notions of linguistic variable and fuzzy logic [1-3], and distinguished the importance of fuzzy truth values as very true, quite true etc., that its are fuzzy subsets of the set of all truth degrees, i. e., its truth-valued are linguistic values of the linguistic truth variable, which are represented by fuzzy sets in the interval $[0,1]$. In 1987, G. Takeuti and S. Titani investigated so-called globalization which can be seen as an interpretation of connective "fully true" [4]. Nguyen Cat Ho and Wolfgang Wechler proposed an algebraic model of Hedge algebra for deal with linguistic information [56]. Since then, there existed some importance results on uncertainty information processing with linguistic terms. In 2000, P. Hájek and D. Harmancova adopted A. D. Yashin axioms of the "strong future tense operator" [8] in Gödel logic and obtained a complete axiomatization for logical connective "more or less" [7]. Since then, P. Hájek has discussed logic $\mathrm{BL}_{\mathrm{vt}}$ which is a conservative extension of BL-logic including logical connective "very true", and semantics given by BL-algebras extended by a unary function V interpreting "very true" [9]. In 2006, Vilém Vychodil has introduced a complete axiomatization of unary connectives interpreted by monotone and super diagonal truth functions, so-called truth-depressing hedges [10]. These connectives formalize linguistic hedges likes "slightly true" and "more or less". Nevertheless, how far can even this sort of fuzzy logic be captured by standard methods of mathematical logic. Therefore, there some approach which use linguistic assessments take the place of numerical values by means of linguistic variables [11-13]. Moreover, variable values are not numbers but words or sentences in a natural or artificial language. In real uncertainty reasoning and approximate inference, there exist many situations in which the information can not be assessed precisely in a quantitative form but may be in a qualitative one that is description in natural language [14]. For example, when ones try to evaluate "Age", ones tend to apply natural language "slightly young, somewhat young, almost young and very young etc." description. We know these descriptions are generated from modifiers and meta truth values by various linguistic and connectives [15-18]. In these situations, a modifier and meta truth value application is efficient. Moreover, some linguistic modifiers seem difficult to distinguish their boundary sometimes, but their meaning of common using can be understood. According to the above viewpoints, a linguistic truthvalued lattice implication algebra for a valuation domain has been proposed in [19]. As a continuous work of $[19,15,16,17]$, this paper extends latticevalued propositional logic system $\mathrm{LP}(\mathrm{X})[20,21,22$, $23,24]$ to the corresponding linguistic truth-valued lattice value propositional logic system $\ell \mathrm{P}(\mathrm{X})$.

\section{Preliminaries}

First of all, we recall some definitions and results which will be needed.

Definition $2.1{ }^{[25,24]}$ Let $(L, \vee, \wedge, ')$ be a bounded lattice with an ordered-reversing involution ' 
and the universal bounds $\mathrm{O}, \mathrm{I}, \rightarrow: L \times L \rightarrow L$ be a mapping. $\quad(L, \vee, \wedge,, \rightarrow)$ is called a lattice implication algebra if it satisfies the following axioms:

$$
\begin{aligned}
& \left(L_{1}\right) x \rightarrow(y \rightarrow z)=y \rightarrow(x \rightarrow z), \\
& (L 2) x \rightarrow x=I, \\
& (L 3) x \rightarrow y=y^{\prime} \rightarrow x^{\prime}, \\
& (L 4) x \rightarrow y=y \rightarrow x=I \text { imply } x=y, \\
& (L 5)(x \rightarrow y) \rightarrow y=(y \rightarrow x) \rightarrow x, \\
& (L 6)(x \vee y) \rightarrow z=(x \rightarrow z) \wedge(y \rightarrow z), \\
& (L 7)(x \wedge y) \rightarrow z=(x \rightarrow z) \vee(y \rightarrow z),
\end{aligned}
$$

for all $x, y, z \in L$.

Definition $2.2^{[24]}$ Let $L$ be a lattice implication algebra, for all $x, y, z \in L, J \subseteq L$ is said to be a filter of $\mathrm{L}$, if it satisfies the following conditions:

(1) $I \in J$; (2) if $x \in J$ and $x \rightarrow y \in J$, then $y \in J$.

$J \subseteq L$ is said to be a implicative filter of $\mathrm{L}$, if it satisfies the following conditions:

(1) $I \in J$; (2) if $x \rightarrow y \in J$ and $x \rightarrow(y \rightarrow z) \in J$, then $x \rightarrow z \in J$.

Definition 2.3 ${ }^{[18,19]}$ Denote MT $=\{$ True (Tr for short), False (Fa for short) $\}$, which is called as the set of meta truth values. The lattice implication algebra defined on the set of meta truth values is called a meta linguistic truth-valued lattice implication algebra, where $\mathrm{Fa} \prec \mathrm{Tr}$, the operation " '” is defined as: $\mathrm{Tr}$ ' $=\mathrm{Fa}$ and $\mathrm{Fa}=\mathrm{Tr}$, the operation " $\rightarrow$ " is defined as $\rightarrow: M T \times M T \rightarrow M T, x \rightarrow y=x^{\prime} \vee y$.

Definition 2.4 ${ }^{[19]}$ Denote $\mathrm{AD}=\{$ Slightly (S1 for short), Somewhat(So), Rather(Ra), Almost (Al), Exactly(Ex), Quite(Qu), Very(Ve), Highly(Hi), Absolutely $(\mathrm{Ab})\}$, which is called as the set of modifiers. The lattice implication algebra defined on the

chain

$\mathrm{Sl} \prec \mathrm{So} \prec \mathrm{Ra} \prec \mathrm{Al} \prec \mathrm{Ex} \prec \mathrm{Qu} \prec \mathrm{Ve} \prec \mathrm{Hi} \prec \mathrm{Ab}$ is called lattice implication algebra with modifiers if its implication is Łukasiewicz implication.

In the following, denote $L=A D \times M T$. Let

$$
\begin{aligned}
& L_{9}=\left\{a_{1}, a_{2}, a_{3}, a_{4}, a_{5}, a_{6}, a_{7}, a_{8}, a_{9}\right\} \\
& L_{2}=\left\{b_{1}, b_{2}\right\} .
\end{aligned}
$$

We can define two Lukasiewicz lattice implication algebras on them respectively, and still denote them as $L_{9}, L_{2}$;

$L_{9}: a_{1} \prec a_{2} \prec a_{3} \prec a_{4} \prec a_{5} \prec a_{6} \prec a_{7} \prec a_{8} \prec a_{9}$, $a_{i} \rightarrow_{\left(L_{9}\right)} a_{j}=a_{(9-i+j) \wedge 9}, a^{\prime\left(L_{9}\right)}=a_{i} \rightarrow_{\left(L_{9}\right)} a_{1}$;

$L_{2} \quad: \quad b_{1} \prec b_{2}, b_{1}^{\prime\left(L_{2}\right)}=b_{2} \quad, \quad b_{2}^{\left(L_{2}\right)}=b_{1}$, $b_{i} \rightarrow_{\left(L_{2}\right)} b_{j}=\left\{\begin{array}{l}b_{1}(i=2, j=1) \\ b_{2}(\text { otherwise })\end{array}\right.$.
Definition $2.5^{[19]}$ The lattice implication algebra $\mathrm{L}$ defined above is called a linguistic truth-valued lattice implication algebra generated by $\mathrm{AD}$ and $\mathrm{MT}$, denoted a L-LIA.

Theorem 2.6 ${ }^{[19]}$ The following conclusions hold for any $(x, y) \in L_{18}$.

(1) $(x, y)^{\prime}=\left(x^{\prime}, y^{\prime}\right) ;$ (2) $\left(x^{\prime}, y^{\prime}\right) \geq\left(x^{\prime}, y\right)$ iff $y^{\prime} \geq y$; (3) $\left(x^{\prime}, y^{\prime}\right) \geq(x, y)$ iff $x^{\prime} \geq x$;

(4) The relation between $\left(x^{\prime}, y\right)$ and $\left(x, y^{\prime}\right)$ are shown in Table 1 , where " $\left(x^{\prime}, y\right) / /\left(x, y^{\prime}\right)$ " means that they are incomparable.

Table 1 The relation between $\left(x^{\prime}, y\right)$ and $\left(x, y^{\prime}\right)$ :

\begin{tabular}{|l|l|l|l|}
\hline & $y \succ y^{\prime}$ & $y=y^{\prime}$ & $y=y^{\prime}$ \\
\hline$x \succ x^{\prime}$ & $/ /$ & $\prec$ & $\prec$ \\
\hline$x=x^{\prime}$ & $\succ$ & $=$ & $\prec$ \\
\hline$x \prec x^{\prime}$ & $\succ$ & $\succ$ & $/ /$ \\
\hline
\end{tabular}

\section{The main results of L-LIA}

In what follows let $\mathrm{L}_{2 \mathrm{~N}}$ denote a linguistic truth-valued lattice implication algebra unless otherwise specified.

Firstly, in a $\mathrm{L}_{2 \mathrm{~N}}=\mathrm{L}_{\mathrm{N}} \times \mathrm{L}_{2}, \quad I=\left(a_{N}, b_{2}\right)$ and $O=\left(a_{1}, b_{1}\right)$ the greatest and the smallest element of respectively, we define binary operations $\vee, \wedge, \otimes, \oplus$ as follows:

$\left(a_{i}, b_{m}\right) \vee_{\left(L_{2 N}\right)}\left(a_{j}, b_{n}\right)=\left(a_{i} \vee_{\left(L_{N}\right)} a_{j}, b_{m} \vee_{\left(L_{2}\right)} b_{n}\right)$; $\left(a_{i}, b_{m}\right) \wedge_{\left(L_{2 N}\right)}\left(a_{j}, b_{n}\right)=\left(a_{i} \wedge_{\left(L_{N}\right)} a_{j}, b_{m} \wedge_{\left(L_{2}\right)} b_{n}\right)$; $\left(a_{i}, b_{m}\right) \otimes_{\left(L_{2 N}\right)}\left(a_{j}, b_{n}\right)=\left(\left(a_{i}, b_{m}\right) \rightarrow\left(a_{j}, b_{n}\right)^{\prime}\right)^{\prime} ;$ $\left(a_{i}, b_{m}\right) \oplus_{\left(L_{2 N}\right)}\left(a_{j}, b_{n}\right)=\left(a_{i}, b_{m}\right)^{\prime} \rightarrow\left(a_{j}, b_{n}\right)$ for any $\left(a_{i}, b_{m}\right),\left(a_{j}, b_{n}\right) \in L_{2 N}$.

We now obtain the following results:

Theorem 3.1 Let $\mathrm{L}_{2 \mathrm{~N}}$ be a linguistic truth-valued lattice implication algebra. For any $\left(a_{i}, b_{m}\right),\left(a_{j}, b_{n}\right),\left(a_{p}, b_{h}\right),\left(a_{q}, b_{k}\right) \in L_{2 N}$, then $\left(\left(a_{i}, b_{m}\right) \rightarrow\left(a_{j}, b_{n}\right)\right) \leq\left(\left(a_{p}, b_{h}\right)\right.$

$$
\left.\rightarrow\left(a_{i}, b_{m}\right)\right) \wedge\left(\left(a_{j}, b_{n}\right) \rightarrow\left(a_{q}, b_{k}\right)\right)
$$

holds.

Proof. Since

$$
\begin{aligned}
& \left(\left(a_{i}, b_{m}\right) \rightarrow\left(a_{j}, b_{n}\right)\right)^{\prime} \rightarrow\left(\left(\left(a_{p}, b_{h}\right)\right.\right. \\
& \left.\left.\rightarrow\left(a_{i}, b_{m}\right)\right) \wedge\left(\left(a_{j}, b_{n}\right) \rightarrow\left(a_{q}, b_{k}\right)\right)\right) \\
& =\left(\left(\left(a_{i}, b_{m}\right) \rightarrow\left(a_{j}, b_{n}\right)\right)^{\prime} \rightarrow\left(\left(a_{p}, b_{h}\right) \rightarrow\left(a_{i}, b_{m}\right)\right)\right) \\
& \wedge\left(\left(\left(a_{i}, b_{m}\right) \rightarrow\left(a_{j}, b_{n}\right)\right)^{\prime} \rightarrow\left(\left(a_{j}, b_{n}\right) \rightarrow\left(a_{q}, b_{k}\right)\right)\right) \\
& \left.=\left(\left(a_{p}, b_{h}\right) \rightarrow\left(\left(\left(a_{i}, b_{m}\right) \rightarrow\left(a_{j}, b_{n}\right)\right)^{\prime} \rightarrow\left(a_{i}, b_{m}\right)\right)\right)\right) \\
& \left.\wedge\left(\left(a_{j}, b_{n}\right) \rightarrow\left(\left(\left(a_{i}, b_{m}\right) \rightarrow\left(a_{j}, b_{n}\right)\right) \rightarrow\left(a_{q}, b_{k}\right)\right)\right)\right) \\
& =\left(\left(a_{p}, b_{h}\right) \rightarrow\left(\left(a_{i}, b_{m}\right)^{\prime} \rightarrow\left(\left(a_{i}, b_{m}\right) \rightarrow\left(a_{j}, b_{n}\right)\right)\right)\right) \\
& \wedge\left(\left(a_{j}, b_{n}\right) \rightarrow\left(\left(a_{q}, b_{k}\right)^{\prime} \rightarrow\left(\left(a_{i}, b_{m}\right) \rightarrow\left(a_{j}, b_{n}\right)\right)\right)\right)
\end{aligned}
$$




$$
\begin{aligned}
& =\left(\left(a_{p}, b_{h}\right) \rightarrow\left(\left(a_{i}, b_{m}\right)^{\prime} \rightarrow\left(\left(a_{j}, b_{n}\right)^{\prime} \rightarrow\left(a_{i}, b_{m}\right)^{\prime}\right)\right)\right) \\
& \wedge\left(\left(a_{q}, b_{k}\right)^{\prime} \rightarrow\left(\left(a_{j}, b_{n}\right) \rightarrow\left(\left(a_{i}, b_{m}\right) \rightarrow\left(a_{j}, b_{n}\right)\right)\right)\right) \\
& =\left(\left(a_{p}, b_{h}\right) \rightarrow I\right) \wedge\left(\left(a_{q}, b_{k}\right)^{\prime} \rightarrow I\right)=I . \\
& \text { Moreover, get } \\
& \left(\left(a_{i}, b_{m}\right) \rightarrow\left(a_{j}, b_{n}\right)\right)^{\prime} \leq\left(\left(a_{p}, b_{h}\right) \rightarrow\left(a_{i}, b_{m}\right)\right) \\
& \wedge\left(\left(a_{j}, b_{n}\right) \rightarrow\left(a_{q}, b_{k}\right)\right) .
\end{aligned}
$$

This means the proposition holds.

Theorem 3.2 Let $\mathrm{L}_{2 \mathrm{~N}}$ be a linguistic truth-valued lattice implication algebra.

For any $\left(a_{i}, b_{m}\right),\left(a_{j}, b_{n}\right) \in L_{2 N}$,

then $\left(\left(a_{i}, b_{m}\right) \vee\left(a_{j}, b_{n}\right)\right)=\left(\left(a_{i}, b_{m}\right) \rightarrow\left(a_{j}, b_{n}\right)\right)$

$\rightarrow\left(a_{j}, b_{n}\right)$ holds.

Proof. It follows from the operations " $\vee$ " and

" $\rightarrow \quad$ ", we can get

$\left(a_{i}, b_{m}\right) \vee\left(a_{j}, b_{n}\right)=\left(a_{i} \vee_{\left(L_{N}\right)} a_{j}, b_{m} \vee_{\left(L_{2}\right)} b_{n}\right)$,

$\left(\left(a_{i}, b_{m}\right) \rightarrow\left(a_{j}, b_{n}\right)\right) \rightarrow\left(a_{j}, b_{n}\right)$

$\left.=\left(\left(a_{i} \rightarrow_{\left(L_{N}\right)} a_{j}\right) \rightarrow_{\left(L_{N}\right)} a_{j},\left(b_{m} \rightarrow_{\left(L_{2}\right)} b_{n}\right) \rightarrow_{\left(L_{2}\right)} b_{n}\right)\right)$.

Since $a_{i}, a_{j} \in L_{N}, b_{m}, b_{n} \in L_{2}$, and $L_{N}, L_{2}$ are

chains. Thus, we have

$a_{i} \vee_{\left(L_{N}\right)} a_{j}=\left(a_{i} \rightarrow_{\left(L_{N}\right)} a_{j}\right) \rightarrow_{\left(L_{N}\right)} a \quad$ and

$b_{m} \vee_{\left(L_{2}\right)} b_{n}=\left(b_{m} \rightarrow_{\left(L_{2}\right)} b_{n}\right) \rightarrow_{\left(L_{2}\right)} b_{n}$,

it follows that

$\left(\left(a_{i}, b_{m}\right) \vee\left(a_{j}, b_{n}\right)\right)=\left(\left(a_{i}, b_{m}\right) \rightarrow\left(a_{j}, b_{n}\right)\right)$

$\rightarrow\left(a_{j}, b_{n}\right)$ holds.

Similarly, we can $\left(a_{i}, b_{m}\right) \wedge\left(a_{j}, b_{n}\right)=\left(\left(a_{i}, b_{m}\right)^{\prime} \vee\left(a_{j}, b_{n}\right)^{\prime}\right)^{\prime}$.

get

Theorem 3.3 Let $\mathrm{L}_{2 \mathrm{~N}}$ be a linguistic truth-valued lattice implication algebra. If $a_{i} \leq a_{j}$ or $b_{m} \leq b_{n}$ for any $\left(a_{i}, b_{m}\right),\left(a_{j}, b_{n}\right) \in L_{2 N}$, then

$\left(a_{i}, b_{m}\right) \leq\left(a_{i}, b_{n}\right)$ and $\left(a_{i}, b_{m}\right) \leq\left(a_{j}, b_{m}\right)$ hold.

Theorem 3.4 Let $\mathrm{L}_{2 \mathrm{~N}}$ be a linguistic truth-valued lattice implication algebra, for any $\left(a_{i}, b_{m}\right),\left(a_{j}, b_{n}\right),\left(a_{p}, b_{h}\right) \in L_{2 N}$. Then

(1) $\left(a_{i}, b_{m}\right) \rightarrow\left(a_{j}, b_{n}\right) \geq\left(a_{i}, b_{m}\right)^{\prime} \vee\left(a_{j}, b_{n}\right)$;

(2) $\left(a_{j}, b_{n}\right) \rightarrow\left(a_{i}, b_{m}\right)=\left(a_{p}, b_{h}\right) \rightarrow\left(a_{i}, b_{m}\right)$ if and only if $a_{j}=a_{p}$ and $b_{n}=b_{h}$ if and only if $\left(a_{i}, b_{m}\right) \rightarrow\left(a_{j}, b_{n}\right)=\left(a_{i}, b_{m}\right) \rightarrow\left(a_{p}, b_{h}\right)$.

Theorem 3.5 Let $\mathrm{L}_{2 \mathrm{~N}}$ be a linguistic truth-valued LIA, for any $\left(a_{i}, b_{m}\right),\left(a_{j}, b_{n}\right) \in L_{2 N}$. Then

$$
\left(\left(a_{i}, b_{m}\right) \rightarrow\left(a_{j}, b_{n}\right)\right) \vee\left(\left(a_{j}, b_{n}\right) \rightarrow\left(a_{i}, b_{m}\right)\right)=I \text {. }
$$

Proof.Since

$\left(\left(a_{i}, b_{m}\right) \rightarrow\left(a_{j}, b_{n}\right)\right) \vee\left(\left(a_{j}, b_{n}\right) \rightarrow\left(a_{i}, b_{m}\right)\right)$

$=\left(a_{i} \rightarrow_{L_{N}} a_{j}, b_{m} \rightarrow_{L_{2}} b_{n}\right)$

$\vee\left(a_{j} \rightarrow_{L_{N}} a_{i}, b_{n} \rightarrow_{L_{2}} b_{m}\right)$

$=\left(a_{(N-i+j) \wedge N}, b_{(2-m+n) \wedge 2}\right)$

$\vee\left(a_{(N-j+i) \wedge N}, b_{(2-n+m) \wedge 2}\right)$

$=\left(a_{(N-i+j) \wedge N} \vee a_{(N-j+i) \wedge}\right.$,

$$
\begin{aligned}
& \left.b_{(2-m+n) \wedge 2} \vee b_{(2-n+m) \wedge 2}\right) \\
= & \left(a_{((N-i+j) \wedge N) \vee((N-j+i) \wedge N),},\right. \\
& \left.b_{((2-m+n) \wedge 2) \vee((2-n+m) \wedge 2)}\right) \\
= & \left(a_{N}, b_{2}\right)=I .
\end{aligned}
$$

Hence the proposition is proved.

Theorem 3.6 Let $\mathrm{L}_{2 \mathrm{~N}}$ be a linguistic truth-valued LIA, for any $\left(a_{i}, b_{m}\right),\left(a_{j}, b_{n}\right),\left(a_{k}, b_{h}\right) \in L_{2 N}$. Then

$$
\begin{aligned}
&(\left.\left(a_{i}, b_{m}\right) \rightarrow\left(a_{k}, b_{h}\right)\right) \rightarrow\left(\left(a_{j}, b_{n}\right) \rightarrow\left(a_{k}, b_{h}\right)\right) \\
&=\left(a_{j}, b_{n}\right) \rightarrow\left(\left(a_{i}, b_{m}\right) \vee\left(a_{k}, b_{h}\right)\right) . \\
& \text { Proof.Since } \\
&\left(\left(a_{i}, b_{m}\right) \rightarrow\left(a_{k}, b_{h}\right)\right) \rightarrow\left(\left(a_{j}, b_{n}\right) \rightarrow\left(a_{k}, b_{h}\right)\right) \\
&=\left(a_{j}, b_{n}\right) \rightarrow\left(\left(\left(a_{i}, b_{m}\right) \rightarrow\left(a_{k}, b_{h}\right)\right) \rightarrow\left(a_{k}, b_{h}\right)\right) \\
&=\left(a_{j}, b_{n}\right) \rightarrow\left(\left(a_{i} \rightarrow_{L_{N}} a_{k}\right) \rightarrow_{L_{N}} a_{k},\right. \\
&\left.\left.\left(b_{m} \rightarrow_{L_{2}} b_{h}\right) \rightarrow_{L_{2}} b_{h}\right)\right) \\
&=\left(a_{j}, b_{n}\right) \rightarrow\left(a_{i} \vee_{L_{N}} a_{k}, b_{m} \vee_{L_{2}} b_{h}\right) \\
&=\left(a_{j}, b_{n}\right) \rightarrow\left(\left(a_{i}, b_{m}\right) \vee\left(a_{k}, b_{h}\right)\right)
\end{aligned}
$$

Therefore, we have

$$
\begin{aligned}
& \left(\left(a_{i}, b_{m}\right) \rightarrow\left(a_{k}, b_{h}\right)\right) \rightarrow\left(\left(a_{j}, b_{n}\right) \rightarrow\left(a_{k}, b_{h}\right)\right) \\
& \quad=\left(a_{j}, b_{n}\right) \rightarrow\left(\left(a_{i}, b_{m}\right) \vee\left(a_{k}, b_{h}\right)\right) .
\end{aligned}
$$

We can get the following Theorem 3.7 and Theorem 3.8 by the properties of LIA and operators.

Theorem 3.7 Let $\mathrm{L}_{2 \mathrm{~N}}$ be a linguistic truth-valued LIA, for any $\left(a_{i}, b_{m}\right),\left(a_{j}, b_{n}\right) \in L_{2 N}$. Then the following statements hold:

(1) $\left(a_{i}, b_{m}\right) \vee\left(a_{j}, b_{n}\right) \geq\left(a_{i}, b_{m}\right) \quad$ or $\left(a_{i}, b_{m}\right) \vee\left(a_{j}, b_{n}\right) \geq\left(a_{j}, b_{n}\right)$;

(2) $\quad\left(a_{i}, b_{m}\right) \wedge\left(a_{j}, b_{n}\right) \leq\left(a_{i}, b_{m}\right) \quad$ or $\left(a_{i}, b_{m}\right) \wedge\left(a_{j}, b_{n}\right) \leq\left(a_{j}, b_{n}\right)$.

Theorem 3.8 Let $\mathrm{L}_{2 \mathrm{~N}}$ be a linguistic truth-valued LIA, for any $\left(a_{i}, b_{m}\right),\left(a_{j}, b_{n}\right),\left(a_{q}, b_{k}\right)$, $\left(a_{p}, b_{h}\right) \in L_{2 N}$. Then the following statements hold:

(1) $\left(a_{i}, b_{m}\right) \otimes\left(a_{j}, b_{n}\right)=\left(a_{j}, b_{n}\right) \otimes\left(a_{i}, b_{m}\right)$, $\left(a_{i}, b_{m}\right) \oplus\left(a_{j}, b_{n}\right)=\left(a_{j}, b_{n}\right) \oplus\left(a_{i}, b_{m}\right) ;$

(2) $\left(a_{i}, b_{m}\right) \leq\left(a_{j}, b_{n}\right)$ and

$$
\left(a_{p}, b_{h}\right) \leq\left(a_{q}, b_{k}\right) \text { imply }
$$

$\left(a_{i}, b_{m}\right) \otimes\left(a_{p}, b_{h}\right) \leq\left(a_{j}, b_{n}\right) \otimes\left(a_{q}, b_{k}\right)$;

$\left(a_{i}, b_{m}\right) \otimes\left(a_{p}, b_{h}\right) \leq\left(a_{j}, b_{n}\right) ;$

(4) $\quad\left(a_{i}, b_{m}\right) \otimes\left(a_{1}, b_{1}\right)=\left(a_{1}, b_{1}\right)$

$\left(a_{i}, b_{m}\right) \oplus\left(a_{1}, b_{1}\right)=\left(a_{i}, b_{m}\right)$;

$$
\left(a_{i}, b_{m}\right) \otimes\left(a_{N}, b_{2}\right)=\left(a_{i}, b_{m}\right)
$$

$\left(a_{i}, b_{m}\right) \oplus\left(a_{N}, b_{2}\right)=\left(a_{N}, b_{2}\right)=I$;

(6) $\left(\left(a_{i}, b_{m}\right) \otimes\left(a_{j}, b_{n}\right)\right)^{\prime}=\left(a_{i}, b_{m}\right)^{\prime} \oplus\left(a_{j}, b_{n}\right)^{\prime}$, $\left(\left(a_{i}, b_{m}\right) \oplus\left(a_{j}, b_{n}\right)\right)^{\prime}=\left(a_{i}, b_{m}\right)^{\prime} \otimes\left(a_{j}, b_{n}\right)^{\prime}$. 


\section{The semantic of linguistic truth- valued lattice values propositional logic system $\ell P(X)$}

Language Let $L^{*}=\left(L, \vee, \wedge,{ }^{\prime}, \rightarrow\right)$ be a Linguistic Truth-Valued LIA, $L=L_{N} \times L_{2}$.

The symbols in $\ell P(X)$ are

(1) the set of propositional variable:

$X=\{p, q, r, \ldots$.

(2) the set of constants: L;

(3) logical connectives: $\rightarrow$, ;

(4) auxiliary symbols: $),($.

The set $F$ of formula of $\ell \mathrm{P}(\mathrm{X})$ is the least set $\mathrm{Y}$ satisfying the following conditions:

(1) $X \subseteq Y$; (2) $L \subseteq Y$;

(3) if $\left(a_{i}, b_{m}\right),\left(a_{j}, b_{n}\right) \in Y$, then

' $\left(a_{i}, b_{m}\right), \rightarrow\left(a_{i}, b_{m}\right),\left(a_{j}, b_{n}\right) \in Y$.

In the following, we denote $\left(a_{i}, b_{m}\right)$ as $\left(a_{i}, b_{m}\right)^{\prime}$ and $\rightarrow\left(a_{i}, b_{m}\right),\left(a_{j}, b_{n}\right)$ as $\left(a_{i}, b_{m}\right) \rightarrow\left(a_{j}, b_{n}\right)$.

Definition 4.1 The free $T^{*}$ algebra of the set $X$ of the propositional variable is said to be the propositional algebra $\ell \mathrm{P}(\mathrm{X})$ of the linguistic truthvalued lattice value propositional calculus system and denote by $\ell P(X)$ if it satisfies the following conditions: (1) $T^{*}=L^{*} \cup\{, \rightarrow\}$ be a type; (2) for any $(\alpha, \beta) \in L, \operatorname{ar}(\alpha, \beta)=0, \operatorname{ar}()=1$, $\operatorname{ar}(\rightarrow)=2$.

Definition 4.2 A mapping $\varphi: \ell P(X) \rightarrow L$ is called a valuation of $\ell \mathrm{P}(\mathrm{X})$, if it is a $\mathrm{T}$-homomorphism.

Since $\ell P(X)$ is a free $T$-algebra on $X$, there exist only one valuation $\psi$ such that $\left.\varphi\right|_{X}=\psi$ foe any mappin-g $\psi: X \rightarrow L^{*}$.

Let $L^{*}$ be a complete lattice, $F_{L^{*}}(Y)$ is the set of all $L^{*}$-type fuzzy sets in $\mathrm{Y}$.

Corollary 4.3 Let $f: \ell P(X) \rightarrow L^{*}$ be a mapping, then $f$ is a valuation of $\ell \mathrm{P}(\mathrm{X})$ if and only if it satisfies:

(1) $f(\alpha, \beta)=(\alpha, \beta)$ for any $(\alpha, \beta) \in L^{*}$;

(2) $f\left(\left(a_{i}, b_{m}\right) \rightarrow\left(a_{j}, b_{n}\right)\right)$

$=f\left(a_{i}, b_{m}\right) \rightarrow f\left(a_{j}, b_{n}\right)$ for any formula $p=\left(a_{i}, b_{m}\right)$ and $q=\left(a_{j}, b_{n}\right)$;

(3) $f\left(\left(a_{i}, b_{m}\right)^{\prime}\right)=\left(f\left(a_{i}, b_{m}\right)\right)^{\prime}$ for any formula $p=\left(a_{i}, b_{m}\right)$.

Proof. " $\Rightarrow$ ": Suppose $f$ is a valuation of $\ell \mathrm{P}(\mathrm{X})$, then (1), (2) and (3) are hold by Definition4.3.

“ $\Leftarrow$ ": Since $\left(a_{i}, b_{m}\right) \vee\left(a_{j}, b_{n}\right)$

$$
\begin{aligned}
& \quad=\left(\left(a_{i}, b_{m}\right) \rightarrow\left(a_{j}, b_{n}\right)\right) \rightarrow\left(a_{j}, b_{n}\right) \\
& f\left(\left(a_{i}, b_{m}\right) \rightarrow\left(a_{j}, b_{n}\right)\right) \\
& \quad=f\left(a_{i}, b_{m}\right) \rightarrow f\left(a_{j}, b_{n}\right) .
\end{aligned}
$$

and

Thus, we get

$f\left(\left(a_{i}, b_{m}\right) \vee\left(a_{j}, b_{n}\right)\right)$

$=f\left(\left(\left(a_{i}, b_{m}\right) \rightarrow\left(a_{j}, b_{n}\right)\right) \rightarrow\left(a_{j}, b_{n}\right)\right)$

$=\left(f\left(a_{i}, b_{m}\right) \rightarrow f\left(a_{j}, b_{n}\right)\right) \rightarrow f\left(a_{j}, b_{n}\right)$

$=f\left(a_{i}, b_{m}\right) \vee f\left(a_{j}, b_{n}\right)$.

Similarly, we can get

$f\left(\left(a_{i}, b_{m}\right) \wedge\left(a_{j}, b_{n}\right)\right)=f\left(a_{i}, b_{m}\right) \wedge f\left(a_{j}, b_{n}\right)$.

Hence $f$ is a T-homomorphism, i.e., $f$ is a valuation of $\ell \mathrm{P}(\mathrm{X})$. This completes the proof.

Definition 4.4 Let $A \in F_{L^{*}}(F), \varphi$ is a valuation of $\ell P(X)$. It is called that $\varphi$ satisfies $\mathrm{A}$ if

$A\left(a_{i}, b_{m}\right) \leq \varphi\left(a_{i}, b_{m}\right)$ for any $\left(a_{i}, b_{m}\right) \in F$. A is called satisfiable if there exists a valuation $\varphi$, which satisfies A.

Definition 4.5 Let $A \in F_{L^{*}}(F),\left(a_{i}, b_{m}\right) \in F$, $(\alpha, \beta) \in L^{*} .\left(a_{i}, b_{m}\right)$ is called semantically implied from A with truth value level $(\alpha, \beta)$ if $\varphi\left(a_{i}, b_{m}\right) \geq(\alpha, \beta)$ for any valuation $\varphi$, which satisfies $\mathrm{A}$, of $\ell \mathrm{P}(\mathrm{X})$, and denote as $A \mid={ }_{\alpha}\left(a_{i}, b_{m}\right)$. $\left(a_{i}, b_{m}\right)$ is called valid with truth value level $(\alpha, \beta)$ and denoted by $\mid={ }_{(\alpha, \beta)}\left(a_{i}, b_{m}\right)$, if $\varphi\left(a_{i}, b_{m}\right) \geq(\alpha, \beta)$ for any $\varphi$ valuation of $\ell P(X)$. $\left(a_{i}, b_{m}\right)$ is called a valid formula if $={ }_{\left(a_{N}, b_{2}\right)} \cdot\left(a_{i}, b_{m}\right)$ is called $(\alpha, \beta)$-valid in $\mathrm{A}$ if $(\alpha, \beta)=\wedge\left\{\varphi\left(a_{i}, b_{m}\right) \mid \varphi\right.$ is a valuation and satisfies A $\}$.

Definition 4.6 Let $\left(a_{i}, b_{m}\right) \quad$ and $\left(a_{j}, b_{n}\right) \in \ell P(X)$.

$\left(a_{i}, b_{m}\right),\left(a_{j}, b_{n}\right)$ are called equivalent if $\varphi\left(a_{i}, b_{m}\right)$ $=\varphi\left(a_{j}, b_{n}\right)$ for any valuation $\varphi$ of $\ell \mathrm{P}(\mathrm{X})$, deno-ted by $\left(a_{i}, b_{m}\right)=\left(a_{j}, b_{n}\right)$.

Definition 4.7 $A \in F_{L^{*}}(\ell P(X))$ is said to be closed if it satisfies the following conditions: (1) $A\left(\left(a_{i}, b_{m}\right) \rightarrow\left(a_{j}, b_{n}\right)\right) \otimes A\left(a_{i}, b_{m}\right) \leq A\left(a_{j}, b_{n}\right)$;

(2) $\quad(\alpha, \beta) \rightarrow A\left(a_{i}, b_{m}\right) \leq A\left((\alpha, \beta) \rightarrow\left(a_{i}, b_{m}\right)\right)$ for any $\left(a_{i}, b_{m}\right),\left(a_{j}, b_{n}\right) \in \ell P(X),(\alpha, \beta) \in L^{*}$.

Definition 4.8 Let

$X_{n}=\left\{\left(a_{1}, b_{1}\right),\left(a_{2}, b_{2}\right), \ldots,\left(a_{n}, b_{n}\right)\right\}$ and $\omega\left(\left(a_{1}, b_{1}\right),\left(a_{2}, b_{2}\right), \ldots,\left(a_{n}, b_{n}\right)\right) \in \ell P(X)$, for any element $\varpi\left(\left(a_{1}, b_{1}\right),\left(a_{2}, b_{2}\right), \ldots,\left(a_{n}, b_{n}\right)\right)$ of $\overline{\ell P(X)}$ and $\omega\left(\left(a_{1}, b_{1}\right),\left(a_{2}, b_{2}\right), \ldots,\left(a_{n}, b_{n}\right)\right)$

$$
=\varpi\left(\left(a_{1}, b_{1}\right),\left(a_{2}, b_{2}\right), \ldots,\left(a_{n}, b_{n}\right)\right) .
$$

Define a mapping $f_{\bar{\sigma}}: L^{*^{n}} \rightarrow L^{*}, f_{\sigma}$ is called a truth value function of $L^{*^{n}}$ if it satisfies for any 
$\left(\left(\alpha_{1}, \beta_{1}\right),\left(\alpha_{2}, \beta_{2}\right), \ldots,\left(\alpha_{n}, \beta_{n}\right)\right) \in L^{*^{n}}$, there exists a valuation $\varphi$ of $\ell \mathrm{P}(\mathrm{X})$ such that $\varphi\left(a_{i}, b_{m}\right)=\left(\alpha_{i}, \beta_{m}\right)(1 \leq i \leq N, 1 \leq m \leq 2)$, $f_{\bar{\sigma}}\left(\left(\alpha_{1}, \beta_{1}\right),\left(\alpha_{2}, \beta_{2}\right), \ldots,\left(\alpha_{n}, \beta_{n}\right)\right)$

$=\varphi\left(\omega\left(\left(a_{1}, b_{1}\right),\left(a_{2}, b_{2}\right), \ldots,\left(a_{n}, b_{n}\right)\right)\right)$

We can easy to verify the following results by definitions and properties of LIA.

Theorem 4.9 Let $L^{*}$ be a linguistic truth value LIA, for any $\left(a_{i}, b_{m}\right),\left(a_{j}, b_{n}\right),\left(a_{p}, b_{k}\right) \in L^{*}$, then $\left(\left(a_{i}, b_{m}\right) \rightarrow\left(a_{j}, b_{n}\right)\right) \rightarrow\left(\left(a_{i}, b_{m}\right) \rightarrow\left(a_{k}, b_{p}\right)\right)$ $=\left(a_{i}, b_{m}\right) \wedge\left(a_{j}, b_{n}\right) \rightarrow\left(a_{k}, b_{p}\right)$.

Theorem 4.10 Let $L^{*}$ be a linguistic truth value LIA, for any $\left(a_{i}, b_{m}\right),\left(a_{j}, b_{n}\right),\left(a_{p}, b_{k}\right) \in L^{*}$, then $\left(\left(a_{i}, b_{m}\right) \rightarrow\left(a_{j}, b_{n}\right)\right) \rightarrow\left(\left(a_{i}, b_{m}\right) \rightarrow\left(a_{k}, b_{p}\right)\right)$ $\geq\left(a_{j}, b_{n}\right) \rightarrow\left(a_{k}, b_{p}\right)$ holds.

Theorem 4.11 Let $L^{*}$ be a linguistic truth value LIA, for any $\left(a_{i}, b_{m}\right),\left(a_{j}, b_{n}\right),\left(a_{p}, b_{k}\right) \in \ell P(X)$, $\left(a_{i}, b_{m}\right)^{2}=\left(a_{i}, b_{m}\right) \otimes\left(a_{i}, b_{m}\right)$ $\left(a_{i}, b_{m}\right)^{t+1}=\left(a_{i}, b_{m}\right)^{t} \otimes\left(a_{i}, b_{m}\right)$.

Then $\left(\left(a_{i}, b_{m}\right)^{t} \rightarrow\left(\left(a_{j}, b_{n}\right)\right.\right.$ $\left.\left.\rightarrow\left(a_{k}, b_{p}\right)\right)\right) \rightarrow\left(\left(a_{i}, b_{m}\right)^{s} \rightarrow\left(a_{k}, b_{p}\right)\right)$ $\rightarrow\left(\left(a_{i}, b_{m}\right)^{t+s} \rightarrow\left(a_{k}, b_{p}\right)\right)$ is a valid formula, for any $t, s \in N^{+}$.

Theorem 4.12 The valuation of $\ell \mathrm{P}(\mathrm{X})$ is closed.

Corollary 4.13 The valuation of $\ell_{18} \mathrm{P}(\mathrm{X})$ is closed.

$$
\text { Let } \bar{F}=F /=\left\{\overline{\left(a_{i}, b_{m}\right)} \mid \quad\left(a_{i}, b_{m}\right) \in F\right\} \text {, }
$$
where $\left(a_{i}, b_{m}\right)=\left\{\left(a_{j}, b_{n}\right) \mid\left(a_{j}, b_{n}\right) \in F\right.$, $\left.\left(a_{i}, b_{m}\right)=\left(a_{j}, b_{n}\right)\right\}$. It can be proved that $=$ is a congruence relation on $\bar{F}$. Hence, define operations $\vee, \underline{\wedge}, \rightarrow$ on $F$ as follows:

$\left.\underline{\left(\left(a_{i}, b_{m}\right)\right.}\right)^{\prime}=\overline{\left(a_{i}, b_{m}\right)^{\prime}}$ $\underline{\left(a_{i}, b_{m}\right)} \rightarrow \overline{\left(a_{j}, b_{n}\right)}=\overline{\left(a_{i}, b_{m}\right) \rightarrow\left(a_{j}, b_{n}\right)}$ $\underline{\left(a_{i}, b_{m}\right)} \vee \underline{\left(a_{j}, b_{n}\right)}=\underline{\left(a_{i}, b_{m}\right) \vee\left(a_{j}, b_{n}\right)}$ $\left(a_{i}, b_{m}\right) \wedge \overline{\left(a_{j}, b_{n}\right)} \equiv \overline{\left(a_{i}, b_{m}\right) \wedge\left(a_{j}, b_{n}\right)}$.

Let $\overline{\ell P(X)}=(\bar{F}, \vee, \wedge,, \rightarrow)$, it is easy proved the following theorem 4.14 , Theorem 4.15 and Theorem 4.16.

Theorem 4.14 Let $\overline{\ell P(X)}=\left(\bar{F}, \vee, \wedge,{ }^{\prime}, \rightarrow\right)$ is lattice implication algebra.

Theorem $\quad 4.15 \quad(1)$ If $\varphi$ is a valuation, $\quad \bar{\varphi}: \overline{\ell P(X)} \rightarrow L^{*} \quad \underline{\text { satisfies }}$ $\bar{\varphi}\left(\overline{\left(a_{i}, b_{m}\right)}\right)=\varphi\left(a_{i}, b_{m}\right)$ for any $\left(a_{i}, b_{m}\right) \in \overline{\ell P(X)}$, then is a lattice implication epimorphism;

(2) Let $\bar{\varphi}: \overline{\ell P(X)} \rightarrow L^{*}$ is a lattice implication homomorphism and satisfies $\bar{\varphi}(\overline{(\alpha, \beta)})=(\alpha, \beta)$ for

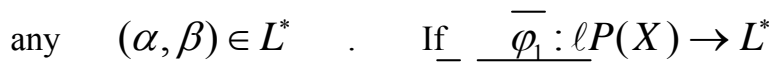
satisfies $\varphi_{1}\left(a_{i}, b_{m}\right)=\bar{\varphi}\left(\overline{\left(a_{i}, b_{m}\right)}\right)$ for any $\left(a_{i}, b_{m}\right) \in L^{*}$. Then $\varphi_{L}$ is a valuation of $\ell \mathrm{P}(\mathrm{X})$.

Theorem 4.16 For any $\bar{\omega}, \omega_{1} \in \overline{\ell P(X)}$, if $f_{\bar{\omega}}=f_{\overline{\omega_{1}}}$ then $\bar{\omega}=\overline{\omega_{1}}$.

\section{Further research}

Future research will focus on the structure of linguistic truth-valued LIA and their resolution procedures based on linguistic truth-valued LIA and the construction method of reasonable linguistic truth-valued LIA.

\section{Acknowledgment}

This work is partially supported by National Nature Science Foundation of China (Grant No. 60474022) and the specialized Research Fund for the Doctoral Program of Higher Education of China (Grant No. 20060613007).

\section{References}

[1] L. A. Zadeh, Fuzzy logic and approximate reasoning. Sythese, 30:407-428, 1975.

[2] L. A. Zadeh, A theory of approximate reasoning. In: R. R. Yager, S. Ovchinnikov, R. M. Tong, $H$. T. Nguyen (Eds.), Fuzzy Sets and Applications: Selected papers by L.A. Zadeh, Wiley, New York, pp:367-411, 1987.

[3] L. A. Dadeh, The concepts of a linguistic variable and its applications to approximate reasoning. Part I, Information Sciences, 8:199249, 1975. Part II, Information Sciences, 8:301357, 1975. Part III, Information Sciences, 9:4380, 1975.

[4] G. Takeuti and S. Titani, Globalization of intuitionitic set theory, International Journal of Ann. Pure Appl. Logic, 33:195-211, 1987.

[5] N. C. Ho and W. Wechler, Hedge algebras: on algebraic approach to structure of sets of linguistic truth values, Fuzzy Sets and Systems, Vol. 35:281-293, 1990.

[6] N. C. Ho and W. Wechler, Extended hedge algebras and their application on fuzzy logic, Fuzzy Sets and Systems, 52:259-281, 1992.

[7] P. Hájek and D. Harmancova, A hedge for Gödel fuzzy logic. International Journal Uncertainty Fuzziness Knowledge-Based Systems, 8:495-498, 2000.

[8] A. D. Yashin, A modified neighborhood semantics for strong future tense operator in intuitionis- 
tic propositional logic. In: Abstracts of Logic Colloquium 97, Leeds, England, 1997.

[9] P. Hajek, On very true, Fuzzy Sets and Systems, 124: 329-233, 2001.

[10] Vilém Vychodil, Truth-depressing hedges and BL-logic, Fuzzy Sets and Systems, 157: 2074-2090, 2006.

[11] F. Herrera and L. Martinez, A 2-tuple fuzzy linguistic representation model for computing with words, IEEE Trans. Fuzzy Systems, 8(6): 746-752, 2000.

[12] Van-Nam Huynh and Yoshiteru Nakamori, A satisfactory-Oriented Approach to Multiexpert Decision-Making with Linguistic Assessments, IEEE Trans. On Systems, Man and Cybernetics Part B: Cybernetics, 35(2): 184-196, 2005.

[13] M. Delgado, J. L. Verdegay, and M. A. Vila, Linguistic decision making models, International Journal of Intelligent Systems, 7: 479-492, 1992.

[14] J. Ma, S. Chen and Y. Xu, Fuzzy logic form the viewpoint of machine intelligence, Fuzzy Sets and Systems, 157(5):628-634, 2006.

[15] S. W. Chen, Y. Xu and J. Ma, A linguistic truthvalued uncertainty reasoning model based on Lattice-Valued Logic, Fuzzy system and knowledge discovery, Second International Conference, FSKD2005, LNAI 3613:276-283, 2005.

[16] D. Meng, H. D. Jia and Y. Xu, Framework of Six Linguistic Lattice-Valued Evaluation System, International Conference on Intelligent Systems and Knowledge engineering, 2006.

[17] Z. Pei and Y. Xu, Lattice implication algebra model of a kind of linguistic terms and its inference, Proc. Of the 6th International FLINS conference, 93-98, 2004.

[18] Y. Xu, J. Liu, D. Ruan, and T. T. Lee, On the consistency of rule bases based on LatticeValued first-order logic LF(X), International Journal of Intelligent Systems, 21:399-424, 2006.

[19] Y. Xu, S. W. Chen and J. Ma, Linguistic Truthvalued Lattice Implication Algebra and Its Properties, Proc. of CESA2006 conference, 1413-1418, 2006.

[20] Y. Xu, K. Y. Qin, Lattice-valued propositional logic (I), Journal of Southwest Jiaotong University, 1: 123-128, 1993.

[21] K. Y. Qin and Y. Xu, Lattice-valued propositional logic (II), Journal of Southwest Jiaotong University, 2(1):22-27, 1994.

[22] K. Y. Qin, Y. Xu and Z. M. Song, Some kinds of approximate reasoning based on system LP(X), Fuzzy Systems and Mathematics, 12 (2): 55-60, 1998. (in Chinese)
[23] K. Y. Qin, Study on lattice-valued logic system based on lattice implication algebras and their applications, Ph. D. thesis, Southwest Jiaotong University, 1996.

[24] Y. Xu, D. Ruan, K. Y. Qin and J. Liu, LatticeValued Logic-An alternative approach to treat fuzziness and incomparability, Springer-verlag, 2003.

[25] Y. Xu, Lattice implication algebras, Journal of Southwest Jiaotong University, 28(1):20-27, 1993. ( in Chinese). 